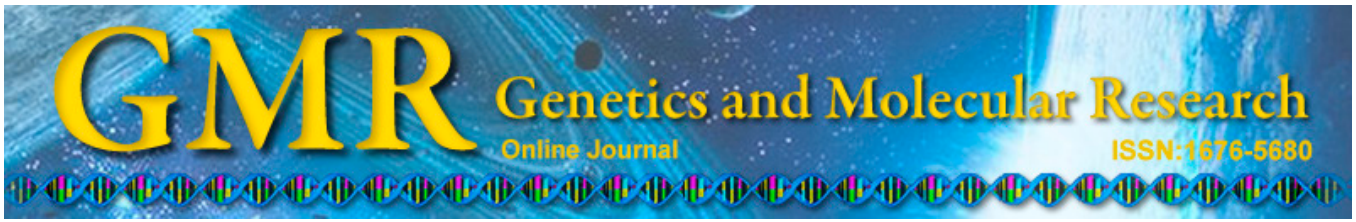

\title{
Effect of the mixture of bone marrow mesenchymal stromal cells and annulus fibrosus cells in repairing the degenerative discs of rabbits
}

\author{
Y.H. Wang1, B. Yang', W.L. Li ${ }^{1}$ and J.M. Li ${ }^{2}$ \\ ${ }^{1}$ Department of Orthopedics, Qingdao Municipal Hospital, Shandong University, \\ Qingdao, Shandong, China \\ ${ }^{2}$ Department of Orthopedics, Qilu Hospital, Shandong University, Jinan, \\ Shandong, China \\ Corresponding author: J.M. Li \\ E-mail: ljmzhi@163.com
}

Genet. Mol. Res. 14 (1): 2365-2373 (2015)

Received May 23, 2014

Accepted October 21, 2014

Published March 27, 2015

DOI http://dx.doi.org/10.4238/2015.March.27.22

ABSTRACT. The aims of this study were to investigate the effect
of a mixture of bone marrow mesenchymal stem cells (BMSCs) and
annulus fibrosus cells (AFCs) in repairing the degenerative discs of
rabbits, and to provide an experimental basis for its clinical application.
BMSCs from rabbits were cultured in vitro and mixed with AFCs.
The animal model of degenerative intervertebral disc was built by
aspirating the nucleus pulposus (L3-4, L4-5, L5-6, and L6-7) through
a posterolateral approach. Mixtures of BMSCs and AFCs (group A),
BMSCs alone (B), or saline (C) were injected into test discs, and
changes evaluated by plain radiographs, magnetic resonance imaging,
and histology. After two weeks, each group showed typical internal
disc disruption; stenosis of the intervertebral space, weakening T2
disc signal, decreased disc height and expression of type II collagen 
and glycosaminoglycan, and fibrosis of the nucleus pulposus. After cell transplantation, the disc heights in groups A and B were regained; however, they continued to decrease in group $\mathrm{C}$. The transplanted cells survived in the discs, proliferated after transplantation, and produced copious matrix. Macroscopic and histological evaluations confirmed structural and nuclear preservation in cell-transplanted discs. The secretions and expressions of Type II collagen and glycosaminoglycan in group A were statistically significant higher than those in group B (Type II collagen, group A $141.6 \pm 5.87$, group B $139.8 \pm 8.65, \mathrm{P}=$ 0.004; glycosaminoglycan, group A $3.008 \pm 0.35$, group B $2.94 \pm 0.29$, $\mathrm{P}=0.003$ ). Expression of type II collagen and glycosaminoglycan was significantly greater in group A than group B. Therefore, cotransplantation of BMSCs and AFCs can restore the extracellular matrix, making this approach superior to transplantation of BMSCs alone, which may be beneficial for the therapy of intervertebral disc degeneration.

Key words: Intervertebral disc degeneration; Tissue engineering repair; Bone marrow mesenchymal stem cell; Annulus fibrosus cell; Co-cultured-transplantation; Rabbit

\section{INTRODUCTION}

Degeneration of the intervertebral discs is strongly implicated as a cause of lower back pain (LBP) (Macintosh and Bogduk, 1991; Freemont, 2009), which is considered to be a public health concern by the global medicine community (Dagenais et al., 2008). Current treatment approaches, based on conservative treatments and operations, can ameliorate the patients' clinical symptoms, but are limited by the concentration on surgical techniques and the use of novel biological solutions e.g. biogels and dynamic stabilization (Schizas et al., 2010; Wang et al., 2010).

Some studies have reported that internal disc disruption (IDD) is accompanied by a decrease in the number of nucleus pulposus cells (NPCs) and annulus fibrosus cells (AFCs), suggesting that methods promoting the regeneration of NPCs and AFCs could be used as effective treatments for IDD (Freemont, 2009; Miyamoto et al., 2010). Harnessing the potential of stem cells is an important strategy for regenerative medicine, as shown by the use of bilaminar coculture pellets of mesenchymal stem cells (MSCs) and NPCs as a cell-based therapy for intervertebral disc regeneration (Allon et al., 2010; Tian and Qi, 2010). In our previous study, we found that co-culture of bone marrow mesenchymal stem cells (BMSCs) and AFCs can alter cell proliferation and the expression of glycosaminoglycan mRNA and type II collagen (Kim et al., 2010).

Together, these promising findings have motivated the present study. The objectives were to investigate the effect of a mixture of BMSCs and AFCs in repairing the degenerative discs of rabbits, and to provide an experimental basis for its clinical application. 


\title{
MATERIAL AND METHODS
}

\begin{abstract}
Animals
Sixty New Zealand white rabbits, aged 6-months-old of either gender and weighing 1-1.5 kg, were used in this study, and were purchased from the Experimental Animal Center of Qingdao City, Shandong Province, China (permission No. SYXK 2013 0811). All animals were raised and procedures conducted in strict accordance with the Animal Ethical Standard, and this study was approved by the Experimental Animal Center of Qingdao City, Shandong Province Ethics Committee.
\end{abstract}

\section{Co-culture of BMSCs and AFCs in vitro}

BMSCs were isolated from animals following the method described previously (Ho et al., 2008). In brief, the femurs and tibias of the rabbits were removed, and muscles and extraosteal tissues were trimmed. The flushed bone marrow cells were centrifuged on a $1.073 \mathrm{~g} /$ $\mathrm{mL}$ Percoll density gradient (Pharmacia, St. Louis, MO, USA). After being washed twice with phosphate-buffered saline (PBS), the cells were seeded into $25-\mathrm{cm}^{2}$ cell culture flasks containing L-Dulbecco's modified Eagle medium (DMEM) supplemented with 10\% fetal bovine serum (FBS) and $1 \%$ penicillin-streptomycin. Finally, the cultures were incubated at $37^{\circ} \mathrm{C}$ in $5 \%$ $\mathrm{CO}_{2}$ for $48 \mathrm{~h}$, and then changed into original medium on the fourth day. After 7-10 days in culture, the cells were detached using trypsin/ethylenediaminetetraaacetic acid (EDTA) $(0.25 \%$ trypsin and $0.02 \%$ EDTA) (Sigma, St. Louis, MO, USA), and concentrated by centrifugation at $1000 \mathrm{rpm}$ for $5 \mathrm{~min}$. The suspended BMSCs were seeded on 24-well plates at a density of 1 x $10^{6}$ cells/well and cultured for two days.

AFCs obtained from the spines of rabbits were cultured according to the method described previously (Pan et al., 2012). Briefly, rabbits were sacrificed, and the spine was removed and placed in culture medium. The annulus fibrosus was excised and the marrow was removed. The annulus fibrosus were isolated, sectioned into small pieces $\left(1 \mathrm{~mm}^{3}\right)$, and digested enzymatically for 45 min with $0.25 \%$ trypsin and $0.03 \%$ collagenase in Hanks' balanced salts solution. After digestion, cells were pelleted at $800 \mathrm{rpm}$ in a Beckman TJ-6 centrifuge for $5 \mathrm{~min}$, the supernatant was removed, and cells were resuspended in $5 \mathrm{~mL}$ of DMEM/ F12 containing $10 \%$ FBS and 100 units/mL penicillin/streptomycin. Cells were dissociated by repeatedly passing them through a narrowed Pasteur pipette, then added to a laminin-coated $35-\mathrm{mm}$ dish, and incubated at $37^{\circ} \mathrm{C}$ and $5 \% \mathrm{CO}_{2}$ for 3 days. All cells were co-cultured in the cell culture media, at a density of $2 \times 10^{5} / \mathrm{cm}^{2}$ with a ratio of $1: 1$, at $37^{\circ} \mathrm{C}$ in $5 \% \mathrm{CO}_{2}$ for $48 \mathrm{~h}$.

\section{Establishment of IDD rabbit models}

Sixty New Zealand white rabbits were anesthetized by auricular vein intravenous injection of $1 \%$ pentobarbital sodium. Rabbits were laid on the stereotaxic apparatus, and 20 $\mu \mathrm{L}$ solution was injected into the longitudinal posterior midline incision. The operation was performed in experimental rabbits via the anterior retroperitoneal approach. The management for the intervertebral discs was as follows: if necessary, the transverse process was destroyed, and the L3-4, L4-5, L5-6, and L6-7 processes were exposed by blunt dissection of paraspinal muscles, and 8-12 mg nucleus pulposus was suctioned along a 5-mm longitudinal posterior 
midline incision; the control group was processed by needle stab without suction. After the surgical procedure, $20 \mathrm{U}$ benzylpenicillin sodium and $0.25 \mathrm{mg}$ streptomycin were administered by injection for prevention of infection. MRI scan was used to evaluate the model and $\mathrm{T} 2<1.10$ was considered as successful symbol. Thirty six rabbits were screened out ( 1 died on the $2^{\text {nd }}$ day postoperation, 1 died on the 7 th day post-operation, and 2 exhibit $\mathrm{T} 2<1$ when examined at 2 weeks post-operation). Among them, 30 rabbits were randomly selected for the following experiments.

\section{Grouping of test subjects}

Animals were randomly divided into four groups (five rabbits in each group): those receiving co-cultures of BMSCs and AFCs, cultures of BMSCs only, injection of physiological saline, and the control (untreated) group.

\section{Magnetic resonance imaging (MRI)}

Following auricular vein intravenous injection of $1 \%$ pentobarbital sodium, animals underwent MRI scans, and the differences between the four groups were measured ( 5 rabbits for each time point at $1 \mathrm{~d}$ before operation, $1 \mathrm{~d}$ before cell injection, and 2 weeks after cell injection). Sagittal T2-weighted images were obtained using the following settings: fast spin echo sequence, $\mathrm{TR}=3500 \mathrm{~ms}, \mathrm{TE}=116 \mathrm{~ms}$, field of view $=1 \mathrm{~mm}$, and section thickness $=1.0 \mathrm{~mm}$. The Pfirrmann classification (Gawri et al., 2011), with grades ranging from I (normal) to V (advanced degeneration), was used to assess the degree of disc degeneration.

\section{Histological and immunohistochemical analysis}

Following cell transplantation, the nucleus pulposus was isolated and used for histological and immunohistochemical analysis. In brief, the sample was and fixed with $0.1 \mathrm{M}$ PBS containing $4 \%$ paraformaldehyde for $24 \mathrm{~h}$ at $4{ }^{\circ} \mathrm{C}$, washed three times for $30 \mathrm{~min}$ in PBS, embedded, sectioned $(5 \mathrm{~mm})$, and mounted on silane-coated slides. The sections were dewaxed, dried overnight, and irradiated with microwaves in $0.01 \mathrm{M}$ sodium citrate buffer, $\mathrm{pH}$ 6.0. After washing with PBS (pH 7.4), the sections were treated with hematoxylin and eosin (H\&E) stain, or with $0.3 \%$ hydrogen peroxide for $30 \mathrm{~min}$, incubated with the corresponding normal serum, and then incubated for $1 \mathrm{~h}$ at $37^{\circ} \mathrm{C}$, with type I collagen antibody $(1: 200)$ and type II collagen antibody (1:200) (bs-8859R, Bioss, Beijing, China). The antibodies were detected with biotinylated secondary antibodies followed by a streptavidin-biotin-peroxidase complex (Vectastain Elite; Vector, Burlingame, CA, USA). The grayscale image was analyzed by the Leica Q550 IW software (Leica Microsystems, Wetzlar, Germany), and grayscale values recorded from 5 individual slices were averaged.

\section{Reverse-transcription polymerase chain reaction (RT-PCR)}

Following digestion of thoracic intervertebral discs with papain supplemented by 50 $\mathrm{mM}$ EDTA and $5 \mathrm{mM}$ L-cysteine, total RNA was extracted from the nucleus pulposus using Trizol reagent (Invitrogen, Carlsbad, CA, USA), and the expression of type II collagen and glycosaminoglycan mRNA was analyzed by RT-PCR. The primers were designed by the Primer5 5.0 software (PREMIER Biosoft International, USA) and synthesized by Invitrogen. 
The primers were as follows:

Glycosaminoglycan Forward primer: 5'-TAACGCCAGCAGCAAGTA-3' Reverse primer: 5'-AGGGTCCGAGAAGTCAGC-3', $\beta$-actin Forward primer: 5'-CGTGCGGGACAT CAAGGA-3' Reverse primer: 5'-AGGAAGGAGGGCTGGAACA-3'.

The RT-PCR experiments for quantitative analysis of changes in type II collagen and glycosaminoglycan expression levels were replicated 3 times, and band density (grey scale) was normalized to that of $\beta$-actin. The reactions were performed using the SYBR PrimeScript RT-PCR kit (Takara Bio, Inc., Otsu, Japan) with an ABI 7500 Real-Time PCR System (Applied Biosystems, Foster City, CA, USA) according to standard protocols. As an internal control, levels of $\beta$-actin were quantified in parallel with the target genes. Crossing threshold $\left(\mathrm{C}_{t}\right)$ values were calculated by the 7500 Fast Real-Time PCR System software using the second derivative maximum method. Data were analyzed using the $2^{-\Delta \Delta C T}$ method. All samples were run in triplicate.

\section{Statistical analysis}

Statistical analyses were conducted using the Statistical Package for Social Sciences System Version 14.0 (SPSS, Chicago, IL, USA). Nonparametric data (Pfirrmann grading and histological grading) were analyzed by the Kruskal-Wallis $H$ test. Gene expression changes at different observation times were analyzed by one-way analysis of variance. A P value $<0.05$ was considered to be statistically significant.

\section{RESULTS}

\section{MRI analysis}

Pfirrmann classification results are shown in Table 1. At two weeks after surgery, the signal intensities on T2-weighted images of most punctured discs were similar, with only a few discs exhibiting a slight decrease, and there was no significant difference in any of the three groups. After injection of cells, the degree of degeneration in the punctured discs was mainly grade II to III according to the Pfirrmann classification in groups $\mathrm{A}, \mathrm{B}$, and $\mathrm{C}$, and there were significant differences between groups A, B, and C (Table 1). In addition, the relative value was estimated by MRI; T2-weighted images of most punctured discs exhibited a different grade or a gradual increase in Pfirrmann grade after needle puncture alone $(\mathrm{P}<0.05)$, which is similar with the results following injection of cells (Table 2).

Table 1. Disc height ratio in all groups after cell transplantation.

\begin{tabular}{lccc}
\hline Group & One day before animal modeling & One day before cell transplantation & Two weeks after cell transplantation \\
\hline A & $1.06 \pm 0.08$ & $0.85 \pm 0.11^{*}$ & $0.96 \pm 0.16^{\mathbf{\Delta}}$ \\
B & $1.12 \pm 0.14$ & $0.81 \pm 0.06^{*}$ & $0.97 \pm 0.11^{\mathbf{\Delta}}$ \\
C & $0.99 \pm 0.15$ & $0.78 \pm 0.13^{*}$ & $0.86 \pm 0.15^{\mathbf{\Delta}}$ \\
D & $1.05 \pm 0.11$ & $1.03 \pm 0.07$ & $1.04 \pm 0.08$ \\
\hline
\end{tabular}

*Comparison with the results from one day before animal modeling; $\mathrm{P}<0.05,{ }^{\mathbf{\Delta}}$ Comparison with the results from one day before cell transplantation; $\mathrm{P}<0.05$, ${ }^{\#}$ Comparison with the results from group $\mathrm{C}$ during the same time point; $\mathrm{P}<0.05,{ }^{\$}$ Comparison with the results from group $\mathrm{B}$ during the same time point; $\mathrm{P}<0.05$. 
Table 2. Comparison of $\mathrm{T} 2$ grades in intervertebral discs between pre- and post-operative time points.

\begin{tabular}{lccc}
\hline Group & One day before animal modeling & One day before cell transplantation & Two weeks after cell transplantation \\
\hline A & $0.81 \pm 0.08$ & $0.65 \pm 0.07^{*}$ & $0.74 \pm 0.16^{\mathbf{\Delta} \text { S }}$ \\
B & $0.79 \pm 0.10$ & $0.62 \pm 0.06^{*}$ & $0.68 \pm 0.11^{\mathbf{\Delta}}$ \\
C & $0.76 \pm 0.13$ & $0.59 \pm 0.04^{*}$ & $0.51 \pm 0.15^{\mathbf{\Delta}}$ \\
D & $0.83 \pm 0.07$ & $0.84 \pm 0.11$ & $0.83 \pm 0.08$ \\
\hline
\end{tabular}

*Comparison with the results from one day before animal modeling; $\mathrm{P}<0.05,{ }^{\wedge}$ Comparison with the results from one day before cell transplantation; $\mathrm{P}<0.05$, ${ }^{\#}$ Comparison with the results from group $\mathrm{C}$ during the same time point; $\mathrm{P}<0.05,{ }^{\$}$ Comparison with the results from group $\mathrm{B}$ during the same time point; $\mathrm{P}<0.05$.

\section{Histological analysis}

At 2 weeks after surgery, histological (H\&E) staining of sagittal sections indicated that the severity of IDD increased gradually. Normal disc sections showed an intact annulus fibrosus, a well-defined border between the annulus fibrosus and nucleus pulposus, and a nucleus pulposus consisting of numerous chondrocyte-like cells. There were significant differences in histological characteristics between control discs and discs examined two weeks after injection of cells: the border between the annulus fibrosus and nucleus pulposus was less distinct than that in the pre-operative normal disc, and there was a loss of nucleus pulposus accompanied by a reduction in the number of chondrocyte-like cells. Cell transplantation in group A improved the IDD more significantly than that in group B.

\section{Protein and mRNA expression}

Post-surgery, immunohistochemical analysis showed decreased type II collagen positive staining in regions of IDD of injection groups $\mathrm{A}, \mathrm{B}$, and $\mathrm{C}$, and there was no significant difference between groups. After cell transplantation, positive type II collagens were upregulated more significantly in group $\mathrm{A}$ than that in group $\mathrm{B}(\mathrm{P}<0.05)$, but were downregulated in group C (Table 3).

\section{Table 3. Comparison of the expression of type II collagen in all groups.}

\begin{tabular}{lccc}
\hline Group & One day before animal modeling & One day before cell transplantation & Two weeks after cell transplantation \\
\hline A & $145.6 \pm 5.87$ & $130.6 \pm 4.19^{*}$ & $141.6 \pm 5.87^{\star \text { As }}$ \\
B & $151.7 \pm 8.63$ & $133.4 \pm 5.35^{*}$ & $139.8 \pm 8.65^{\star *}$ \\
C & $148.3 \pm 7.38$ & $128.9 \pm 6.04^{*}$ & $115.3 \pm 7.47^{\star}$ \\
D & $153.1 \pm 9.65$ & $151.3 \pm 11.04$ & $150.5 \pm 8.94$ \\
\hline
\end{tabular}

*Comparison with the results from one day before animal modeling; $\mathrm{P}<0.05,{ }^{\Delta}$ Comparison with the results from one day before cell transplantation; $\mathrm{P}<0.05$, ${ }^{\#}$ Comparison with the results from group $\mathrm{C}$ during the same time point; $\mathrm{P}<0.05,{ }^{\$} \mathrm{Comparison}$ with the results from group $\mathrm{B}$ during the same time point; $\mathrm{P}<0.05$.

The expression of glycosaminoglycan mRNA was significantly decreased in groups $\mathrm{A}, \mathrm{B}$, and $\mathrm{C}$ in IDD model animals, and there was no significant difference between groups (Table 4). After cell transplantation, the expression of aggrecan mRNA increased more significantly in group $\mathrm{A}$ than that in group $\mathrm{B}(\mathrm{P}<0.05)$, but no change was observed in group $\mathrm{D}$; and aggrecan mRNA was decreased in group $\mathrm{C}$. 
Table 4. Comparison of the expression of glycosaminoglycan mRNA in all groups.

\begin{tabular}{lccc}
\hline Group & One day before animal modeling & One day before cell transplantation & Two weeks after cell transplantation \\
\hline A & $3.17 \pm 0.35$ & $0.27 \pm 0.12^{*}$ & $3.08 \pm 0.35^{\mathbf{A} \text { *s }}$ \\
B & $3.26 \pm 0.19$ & $0.31 \pm 0.09^{*}$ & $2.94 \pm 0.29^{\mathbf{A}}$ \\
C & $3.71 \pm 0.40$ & $0.39 \pm 0.13^{*}$ & $0.18 \pm 0.05^{\mathbf{\Delta}}$ \\
D & $3.58 \pm 0.29$ & $3.40 \pm 0.37$ & $3.47 \pm 0.37$ \\
\hline
\end{tabular}

*Comparison with the results from one day before animal modeling; $\mathrm{P}<0.05,{ }^{\star}$ Comparison with the results from

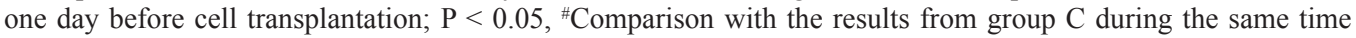
point; $\mathrm{P}<0.05,{ }^{\$}$ Comparison with the results from group $\mathrm{B}$ during the same time point; $\mathrm{P}<0.05$.

\section{DISCUSSION}

More than $20 \%$ of all LBP originates from degenerative change in intervertebral discs (IVDs), leading to a gradual process of cellular loss and degradation of the extracellular matrix (ECM) within the IVDs (Dagenais et al., 2008). Until recently, no study has clarified the precise pathogenesis and pathophysiology of IDD, but it may be considered to be related to factors including heredity, environment, ageing, and so on.

Studies have found that a major cause of LBP-related disabilities may involve biochemical changes in the degenerated disc, including loss of hydration and decreased osmotic pressure, quantitative and distributive changes of collagen, decreased proteoglycan content, and downregulated proteoglycan expression (Yang et al., 2009).

The degenerative pathologies of the intervertebral disc have a remarkable social impact in industrialized countries and can lead to serious disabilities (Gaetani et al., 2008; Ieda et al., 2010). Current treatment options consist of conservative treatments (such as symptomatic pharmacological therapies and physiokinetic therapy) and surgical treatments (intervertebral fusion, total disc replacement, nucleus pulposus replacement, or surgical exeresis) (Mauth et al., 2009; Wang and Campbell, 2009). Recent advances in cell therapy have raised the possibility of regenerating the damaged disc; the autologous disc tissue could be withdrawn, regenerated in vitro, and re-implanted. For example, a study by Yurube et al. (2010) examined whether autologous adipose-derived adult stem cells could improve the quality of an in vitro reconstructed nucleus pulposus tissue in rat.

Various kinds of biological therapeutic methods have been studied in the hope of regenerating the dysfunctional discs in IDD, but the long-term goal is the development of a medical treatment modality focused on a more biological regeneration of the inner nucleus pulposus (NP). In vitro cell co-culture and the endoscopic implantation of an injectable material have been the primary foci of past studies, owing to the limited reproductive ability of intervertebral disc (Zigouris et al., 2011).

MSCs isolated from bone marrow aspirates, on the other hand, provide a nearly unlimited cell source with extremely high proliferation activity and the potential to differentiate into several mesenchymal cell lineages, including chondrogenic differentiation, which suggests the possibility that intradiscal transplantation of MSCs might delay IDD (Clifford et al., 2012). The adipocyte fraction is an important component of IVDs, and its critical role in prevention and treatment of IDD is associated with the integrity of its structure and function (Jim et al., 2011). A three-dimensional (3D) co-culture of NPCs and adipose tissue non-AFCs was assessed in a previously developed alginate 3-D culture system. Morphological investigation of cultured and co-cultured cells was performed using transmission electron microscopy 
and immunofluorescence for collagen type I, aggrecan, CD90, CD34, and vimentin. Results indicated that co-culture of NPCs and non-AFCs improved the quality of the in vitro reconstructed tissue in terms of extracellular matrix production and 3-D cell organization (Gawri et al., 2011). Technological resources are also available for NPC encapsulation intended for regenerating the IVD.

Aggrecan fragmentation and loss is one of the hallmarks of disc degeneration and occurs early in the degenerative process, as well as in normal ageing, and has been commonly used as a marker of disc degeneration (Barbir et al., 2011; Miyamoto et al., 2010). Loss of aggrecan can be due to either an increase in proteinase production or a decrease in aggrecan synthesis, and results in a decreased number of IVDs and the inability to effect biological repair of IDD in advanced stage; this indicates that aglycosaminoglycan are produced by the AFCs could be considered to be a target cell for repair of IDD. In our previous studies, we established a 3-D model of co-culture of BMSCs and AFCs, verified the feasibility of these cultures for clinical use, characterized them as high purity, proliferation, and biologically safe, and demonstrated that BMSCs could promote the expression of type II collagen and proteoglycans in AFCs (Barbir et al., 2011).

In the present study, we constructed an animal model of IDD, and after 2 weeks postoperation, each group developed typical IDD, showing stenosis of the intervertebral space, weakened T2 disc signal, decreased disc height and expression of type II collagen and glycosaminoglycan, and fibrosis of the nucleus pulposus, suggesting that the animal model of IDD was constructed successfully. After cell transplantation, the structure of the inner annulus fibrosus was significantly preserved in the cell-transplanted group, and the boundary between the nucleus and annulus could be clearly visualized; thus, we concluded that the transplanted cells survived in the discs, proliferated after transplantation, and produced ample matrix. The expression of type II collagen and glycosaminoglycan was significantly higher in the BMSC and AFC co-transplanted group than in the BMSC-only group, which is similar to findings of some previous studies (Allen et al., 2009). It is thought that a possible effect of co-cultures of BMSC and AFC transplantation may be to cause the secretion of type II collagen and glycosaminoglycan, which may be beneficial for cytokine transmission and gap junction formation, leading to the repair of IDD.

In conclusion, co-transplantation of BMSCs and AFCs can restore the extracellular matrix, and shows superior transplantation effects than does BMSC transplantation alone, which may be beneficial for the development of therapies for IDD.

\section{REFERENCES}

Allen KD, Griffin TM, Rodriguiz RM, Wetsel WC, et al. (2009). Decreased physical function and increased pain sensitivity in mice deficient for type IX collagen. Arthritis Rheum. 60: 2684-2693.

Allon AA, Aurouer N, Yoo BB, Liebenberg EC, et al. (2010). Structured coculture of stem cells and disc cells prevent disc degeneration in a rat model. Spine J. 10: 1089-1097.

Barbir A, Godburn KE, Michalek AJ, Lai A, et al. (2011). Effects of torsion on intervertebral disc gene expression and biomechanics, using a rat tail model. Spine 36: 607-614.

Clifford DM, Fisher SA, Brunskill SJ, Doree C, et al. (2012). Stem cell treatment for acute myocardial infarction. Cochrane Database System. Rev. 2: CD006536.

Dagenais S, Caro J and Haldeman S (2008). A systematic review of low back pain cost of illness studies in the United States and internationally. Spine J. 8: 8-20.

Freemont AJ (2009). The cellular pathobiology of the degenerate intervertebral disc and discogenic back pain. Rheumatology Oxford, England 48: 5-10. 
Gaetani P, Torre ML, Klinger M, Faustini M, et al. (2008). Adipose-derived stem cell therapy for intervertebral disc regeneration: an in vitro reconstructed tissue in alginate capsules. Tissue Engineering 14: 1415-1423.

Gawri R, Mwale F, Ouellet J, Roughley PJ, et al. (2011). Development of an organ culture system for long-term survival of the intact human intervertebral disc. Spine 36: 1835-1842.

Ho G, Leung VY, Cheung KM and Chan D (2008). Effect of severity of intervertebral disc injury on mesenchymal stem cell-based regeneration. Connect. Tissue Res. 49: 15-21.

Ieda M, Fu JD, Delgado-Olguin P, Vedantham V, et al. (2010). Direct reprogramming of fibroblasts into functional cardiomyocytes by defined factors. Cell 142: 375-386.

Jim B, Steffen T, Moir J, Roughley P, et al. (2011). Development of an intact intervertebral disc organ culture system in which degeneration can be induced as a prelude to studying repair potential. Eur. Spine J. 20: 1244-1254.

Kim JS, Ellman MB, An HS, van Wijnen AJ, et al. (2010). Insulin-like growth factor 1 synergizes with bone morphogenetic protein 7-mediated anabolism in bovine intervertebral disc cells. Arthritis Rheum. 62: 3706-3715.

Macintosh JE and Bogduk N (1991). The attachments of the lumbar erector spinae. Spine (Phila Pa 1976). 16: 783-92.

Mauth C, Bono E, Haas S, Paesold G, et al. (2009). Cell-seeded polyurethane-fibrin structures - a possible system for intervertebral disc regeneration. Eur. Cells Mater. 18: 27-38.

Miyamoto T, Muneta T, Tabuchi T, Matsumoto K, et al. (2010). Intradiscal transplantation of synovial mesenchymal stem cells prevents intervertebral disc degeneration through suppression of matrix metalloproteinase-related genes in nucleus pulposus cells in rabbits. Arthritis Rheum. Ther. 12: R206.

Pan Y, Chu T, Dong S, Hao Y, et al. (2012). Cells scaffold complex for Intervertebral disc Anulus Fibrosus tissue engineering: in vitro culture and product analysis. Mol. Biol. Rep. 39: 8581-8594.

Schizas C, Kulik G and Kosmopoulos V (2010). Disc degeneration: current surgical options. Eur. Cells Mater. 20: 306-315.

Tian W and Qi H (2010). Association between intervertebral disc degeneration and disturbances of blood supply to the vertebrae. Chin. Med. J. 123: 239-243.

Wang BH and Campbell G (2009). Formulations of polyvinyl alcohol cryogel that mimic the biomechanical properties of soft tissues in the natural lumbar intervertebral disc. Spine 34: 2745-2753.

Wang F, Wu XT, Zhuang SY, Wang YT, et al. (2010). Ex vivo observation of human nucleus pulposus chondrocytes isolated from degenerated intervertebral discs. Asian Spine J. 5: 73-81.

Yang F, Leung VY, Luk KD, Chan D, et al. (2009). Mesenchymal stem cells arrest intervertebral disc degeneration through chondrocytic differentiation and stimulation of endogenous cells. Mol. Ther. 17: 1959-1966.

Yurube T, Nishida K, Suzuki T, Kaneyama S, et al. (2010). Matrix metalloproteinase (MMP)-3 gene up-regulation in a rat tail compression loading-induced disc degeneration model. J. Orthop. Res. 28: 1026-1032.

Zigouris A, Batistatou A, Alexiou GA, Pachatouridis D, et al. (2011). Correlation of matrix metalloproteinases-1 and -3 with patient age and grade of lumbar disc herniation. J. Neurosurg. 14: 268-272. 